\title{
Particle Filters for Positioning, Navigation and Tracking
}

\author{
Fredrik Gustafsson, Fredrik Gunnarsson, Niclas Bergman, Urban Forssell, \\ Jonas Jansson, Rickard Karlsson, Per-Johan Nordlund \\ Final version for IEEE Transactions on Signal Processing. \\ Special issue on Monte Carlo methods for statistical signal processing.
}

1

Abstract- A framework for positioning, navigation and tracking problems using particle filters (sequential Monte Carlo methods) is developed. It consists of a class of motion models and a general non-linear measurement equation in position. A general algorithm is presented, which is parsimonious with the particle dimension. It is based on marginalization, enabling a Kalman filter to estimate all position derivatives, and the particle filter becomes low-dimensional. This is of utmost importance for highperformance real-time applications.

Automotive and airborne applications illustrate numerically the advantage over classical Kalman filter based algorithms. Here the use of non-linear models and non-Gaussian noise is the main explanation for the improvement in accuracy.

More specifically, we describe how the technique of map matching is used to match an aircraft's elevation profile to a digital elevation map, and a car's horizontal driven path to a street map. In both cases, real-time implementations are available, and tests have shown that the accuracy in both cases is comparable to satellite navigation (as GPS), but with higher integrity. Based on simulations, we also argue how the particle filter can be used for positioning based on cellular phone measurements, for integrated navigation in aircraft, and for target tracking in aircraft and cars. Finally, the particle filter enables a promising solution to the combined task of navigation and tracking, with possible application to airborne hunting and collision avoidance systems in cars.

\section{INTRODUCTION}

Recursive implementations of Monte Carlo based statistical signal processing [19] are known as particle filters, see [13], [14]. The research has since the paper [21] steadily intensified, see the recent first article collection [13]. The particle filters may be a serious alternative for real-time applications classically approached by model-based Kalman filter techniques [29], [24]. The more non-linear model, or the more non-Gaussian noise, the more potential particle filters have, especially in applications where computational power is rather cheap and the sampling rate is moderate.

\footnotetext{
${ }^{1}$ Fredrik Gustafsson, Fredrik Gunnarsson, Jonas Jansson, Rickard Karlsson and Per-Johan Nordlund are with the Department of Electrical Engineering, Linköping University, 58183 Linköping, Sweden (email: \{fredrik,fred,jansson,rickard,perno\}@isy.liu.se).

Fredrik Gunnarsson is also with Ericsson Radio, Sweden.

Urban Forssell is with NIRA Dynamics AB, 58330 Linköping, Sweden. (urban.forssell@niradynamics.se).

Niclas Bergman is with SaabTech Systems, 17588 Järfälla, Sweden. (email: ncbe@systems.saab.se).

Jonas Jansson is also with Volvo Car Corporation, Sweden.

Rickard Karlsson is also with Saab Dynamics, Sweden.

Per-Johan Nordlund is also with Saab Gripen, Sweden.
}

The paper describes a general framework for a number of applications, where we have implemented the particle filter. The problem areas are

- Positioning, where one's own position is to be estimated. This is a filtering problem rather than a static estimation problem, when an inertial navigation system is used to provide measurements of movement.

- Navigation, where besides the position also velocity, attitude and heading, acceleration and angular rates are included in the filtering problem.

- Target tracking, where another object's position is to be estimated based on measurements of relative range and angles to one's own position.

Another related application fitting this framework, not explicitly included here, is robot localization, see for instance [43], [44]. The problems listed above are related in that they can be described by quite similar state space models, where the state vector contains the position and derivatives of the position. Traditional methods are based on linearized models and Gaussian noise approximations so that the Kalman filter can be applied [1]. Research is focused on how different state coordinates or multiple models can be used to limit the approximations. In contrast to this, the particle filter approximates the optimal solution numerically based on a physical model, rather than applying an optimal filter to an approximate model. A well-known problem with the particle filter is that its performance degrades quickly when the dimension of the state dimension increases. A key theoretical contribution here is to apply marginalization techniques [36], adopted and extended from [12], leading to that the Kalman filter can be used to estimate (or eliminate) all position derivatives, and the particle filter is applied to the part of the state vector containing only the position. Thus, the particle filter dimension is only 2 or 3 , depending on the application, and this is the main step to get real-time high-performance algorithms.

The applications we will describe are:

- Car positioning by map matching. A digital road map is used to constrain the possible positions, where a deadreckoning of wheel speeds is the main external input to the algorithm. By matching the driven path to a road map, a vague initial position (order of km's) can be improved to a meter accuracy. This principle can be used as a supplement to, or even replacement to, GPS (global positioning system). 
- Car positioning by Radio Frequency (RF) measurements. The digital road map above can be replaced by, or supplemented by, measurements from a terrestrial wireless communications system. For hand-over (to transfer a connection from one base station to another) operation, the mobile stations (MS) monitor the received signal powers from a multitude of base stations, and report regularly to the network. These measurements provide a power map which can be used in a similar manner as above. Mobile stations in a near future will moreover provide the possibility of monitoring the traveled distance of the radio signals from a number of base stations [16]. Such measurements can also be utilized in the same manner as with the power measurements.

- Aircraft positioning by map matching or terrain navigation. A Geographical information system contains, among other information, terrain elevation. The aircraft is equipped with sensors such that the terrain elevation can be measured. By map matching, the position can be deducted [5].

- Integrated navigation. The aircraft's Inertial Navigation System (INS) uses dead-reckoning to compute navigation and flight data, i.e. position, velocity, attitude and heading. The INS is regarded as the main sensor for navigation and flight data due to being autonomous and having high reliability [10]. However, small offsets cause drift and its output has to be stabilized. Here, terrain navigation is used today.

- Target tracking. A classical problem in signal processing literature is target tracking, where an IR sensor measures relative angle, or a radar measures relative angle, range and possibly range rate, to the object [4]. For the case of bearings only measuring IR sensor, either the state dynamics or measurement equation is very non-linear depending on the choice of state coordinates, so here the particle filter is particularly promising.

- Combined navigation and tracking. Because the target tracking measurements are relative to one's own platform, positioning is an important sub-problem. Since the sensor introduces a cross-coupling between the problems, a unified treatment is tempting.

- Car collision avoidance is very similar to the target tracking problem, here we are interested in predicting the own car's and other objects' future position, see [40]. Based on the prediction, collision avoidance actions such as warning, braking and steering are undertaken when a collision is likely to happen. In order to have enough time to warn the driver the prediction horizon needs to be quite long. Therefore, utilizing knowledge about road geometry and infrastructure becomes important. One way to improve the prediction of possible maneuvers, is to use information in a digital map. Thus, this is a specific project including all aspects of the problems listed above.

The outline is as follows. We will start with a general framework of models covering all of our applications in Section II. Then, a general algorithm is presented covering all applications, where special attention is paid on practical problems as divergence test, initialization and real-time re- quirements. Each application in the list above is devoted its own section, and conclusions and open questions of general interest are discussed in VIII.

\section{MODELS}

Central for all navigation and tracking applications is the motion model to which various kind of model based filters can be applied. Models which are linear in the state dynamics and non-linear in the measurements are considered:

$$
\begin{aligned}
x_{t+1} & =A x_{t}+B_{u} u_{t}+B_{f} f_{t}, \\
y_{t} & =h\left(x_{t}\right)+e_{t} .
\end{aligned}
$$

Here $x_{t}$ is the state vector, $u_{t}$ measured inputs, $f_{t}$ unmeasured forces or faults, $y_{t}$ the measurements and $e_{t}$ measurement error. We assume independent distributions for $f_{t}, e_{t}$ and $x_{0}$, with known probability densities $p_{e_{t}}, p_{f_{t}}$ and $p_{x_{0}}$, respectively, not necessarily Gaussian. Motion models (1a) are further discussed in Section II-A. These are to a large extent similar in all applications, and standard in the literature. The model (1) takes only movements into account, and we do not attempt to model for instance mechanical dynamics in the platform. That is, (1) have no model parameters. The difference between the applications mainly lies in the availability of measurements. Section II-B provides an extensive list of possible measurement equations (1b).

\section{A. Motion Models}

The signals of primary interest in navigation and tracking applications are related to position, velocity and acceleration as summarized in Table I. Newton's law relates

\begin{tabular}{|l|l|l|l|}
\hline Object & Position & Velocity & Acceleration \\
\hline Own & $p^{(1)}$ & $v^{(1)}$ & $a^{(1)}, \delta a^{(1)}$ acc. bias \\
Other & $p^{(2)}$ & $v^{(2)}$ & - \\
\hline
\end{tabular}

TABLE I

INTERESTING SIGNALS IN NAVIGATION AND TRACKING APPLICATIONS. INDEX (1) AND (2) INDICATES SIGNALS RELATED TO ONE'S OWN AND ANother PlatForm Respectively. All QUANTIties CAN BElONG TO EITHER ONE, TWO OR THREE-DIMENSIONAL SPACES, DEPENDING ON THE APPLICATION.

known and unknown external forces on the platforms to acceleration. From the differential equations $\dot{p}_{t}=v_{t}$ and $\dot{v}_{t}=a_{t}$, we obtain relations like $p_{t}=p_{0}+v_{0} t$ if velocity is assumed constant and $p_{t}=p_{0}+v_{0} t+a_{0} t^{2} / 2$ if acceleration is assumed constant. If we here plug in the sample period $T_{s}$, we get a discrete time model for motion between two consecutive measurements as will be frequently used in the sequel.

Depending on whether the signals are measurable or not, they may be components of either the state vector $x_{t}$ or the input signal $u_{t}$. The ambition here is to discuss models through which the applications are naturally related. 
In specific applications, however, other parameterizations might provide better understanding of design variables and algorithm tuning.

In positioning and navigation applications the signals related to the own platform are of interest. If the velocity $v_{t}^{(1)}$ is assumed measurable (and thus part of the input signal), the state dynamics can be modeled as

$$
p_{t+1}^{(1)}=\underbrace{p_{t}^{(1)}}_{x_{t}}+\underbrace{T_{s} v_{t}^{(1)}}_{B_{u} u_{t}}+\underbrace{T_{s} f_{t}^{(1)}}_{B_{f} f_{t}} .
$$

In several navigation applications, such as airborne, measurements of the acceleration are used instead of velocity. These are typically biased, and the true acceleration can be expressed as

$$
a_{\text {true }, \mathrm{t}}^{(1)}=a_{t}^{(1)}+\delta a_{t}^{(1)}
$$

where $a_{t}^{(1)}$ is the measured acceleration and $\delta a_{t}^{(1)}$ is the bias. The position is extracted by dead-reckoning of the measured acceleration, and therefore the presence of acceleration bias is critical. The natural thing to do is to include the bias in the state vector, and the measured acceleration in the input signal. The resulting motion model is

$$
\begin{gathered}
\left(\begin{array}{c}
p_{t+1}^{(1)} \\
v_{t+1}^{(1)} \\
\delta a_{t+1}^{(1)}
\end{array}\right)=\underbrace{\left(\begin{array}{ccc}
I & T_{s} \cdot I & T_{s}^{2} / 2 \cdot I \\
0 & I & T_{s} \cdot I \\
0 & 0 & I
\end{array}\right)}_{A^{(1)}}\left(\begin{array}{c}
p_{t}^{(1)} \\
v_{t}^{(1)} \\
\delta a_{t}^{(1)}
\end{array}\right) \\
+\underbrace{\left(\begin{array}{c}
T_{s}^{2} / 2 \cdot I \\
T_{s} \cdot I \\
0
\end{array}\right)}_{B_{u}^{(1)}} a_{t}^{(1)}+\underbrace{\left(\begin{array}{c}
T_{s}^{3} / 6 \cdot I \\
T_{s}^{2} / 2 \cdot I \\
T_{s} \cdot I
\end{array}\right)}_{B_{f}^{(1)}} f_{t}^{(1)} .
\end{gathered}
$$

Analogously, a bias in any other measured signal (e.g. a bias in the velocity in Equation (2a)) can be considered by incorporating it in the state equation.

So far, the focus has been on the own platform. In a simple model of the movements of the other platform, the assumption is that its velocity $v^{(2)}$ is subject to an unknown acceleration. This yields

$$
\left(\begin{array}{c}
p_{t+1}^{(2)} \\
v_{t+1}^{(2)}
\end{array}\right)=\underbrace{\left(\begin{array}{cc}
I & T_{s} \cdot I \\
0 & I
\end{array}\right)}_{A^{(2)}}\left(\begin{array}{c}
p_{t}^{(2)} \\
v_{t}^{(2)}
\end{array}\right)+\underbrace{\left(\begin{array}{c}
T_{s}^{2} / 2 \cdot I \\
T_{s} \cdot I
\end{array}\right)}_{B_{f}^{(2)}} f_{t}^{(2)}
$$

In the target tracking literature, a popular choice of motion model is given by the "coordinated turn"-model [4].

When considering tracking of another platform, while moving the own platform, joint navigation and target tracking can be employed. Essentially, the total motion model comprises the motion models $(2 \mathrm{~b})$ and $(2 \mathrm{c})$ :

$$
\begin{aligned}
& \left(\begin{array}{c}
p_{t+1}^{(1)} \\
v_{t+1}^{(1)} \\
\delta a_{t+1}^{(1)} \\
p_{t+1}^{(2)} \\
v_{t+1}^{(2)}
\end{array}\right)=\left(\begin{array}{cc}
A^{(1)} & 0 \\
0 & A^{(2)}
\end{array}\right)\left(\begin{array}{c}
p_{t}^{(1)} \\
v_{t}^{(1)} \\
\delta a_{t}^{(1)} \\
p_{t}^{(2)} \\
v_{t}^{(2)}
\end{array}\right) \\
& +\left(\begin{array}{c}
B_{u}^{(1)} \\
0
\end{array}\right) a_{t}^{(1)}+\left(\begin{array}{cc}
B_{f}^{(1)} & 0 \\
0 & B_{f}^{(2)}
\end{array}\right)\left(\begin{array}{l}
f_{t}^{(1)} \\
f_{t}^{(2)}
\end{array}\right) .
\end{aligned}
$$

\section{B. Measurement Equations}

The main difference between the considered applications is the measurements available. Basically, the measurements are related to the positions of one's own platform $p^{(1)}$ and to the other object $p^{(2)}$. Therefore, the measurement equations can be categorized as depending on $p^{(1)}$ only, or depending on both $p^{(1)}$ and $p^{(2)}$ :

$$
\begin{aligned}
& y_{t}^{(1)}=h^{(1)}\left(p_{t}^{(1)}\right)+e_{t}^{(1)} \\
& y_{t}^{(2)}=h^{(2)}\left(p_{t}^{(1)}, p_{t}^{(2)}\right)+e_{t}^{(2)},
\end{aligned}
$$

where the measurement noise contributions $e_{t}^{(1)}$ and $e_{t}^{(2)}$ are characterized by their distributions. If not explicitly mentioned, a Gaussian distribution is used.

In the studied applications, measurements from at least one of the categories above are available. It is important to note, that any combination of the sensors is possible. The presented applications are just a few examples.

\section{B.1 Measurements of Relative Distance}

As always, any position has to be related to a coordinate system and a reference position. Several types of sensors (e.g. GPS, RF) basically measure the distance relative to that reference point. One possibility is distance measurements of the own position relative to points of known positions $p_{i}, i=1, \ldots, M$, which yields $M$ measurement equations with

$$
h_{a, i}^{(1)}\left(p_{t}^{(1)}\right)=\left|p_{i}-p_{t}^{(1)}\right|, i=1, \ldots, M .
$$

This is also applicable when the position of another object is related to one's own position (e.g. radar, sonar, ultrasound):

$$
h_{b}^{(2)}\left(p_{t}^{(1)}, p_{t}^{(2)}\right)=\left|p_{t}^{(2)}-p_{t}^{(1)}\right|
$$

Some sensors do not measure the relative distance explicitly, but rather a quantity related to the same. One example is sensors that measure the received radio signal power transmitted from a known position $p_{i}$. This received power typically decays as $\sim K_{1} /\left|p_{i}-p_{t}^{(1)}\right|^{\alpha}, \alpha \in[2,5]$, where $K_{1}$ and $\alpha$ depend on the radio environment, antenna characteristics, terrain etc. In a logarithmic scale, the measurements are given by

$$
h_{c, i}^{(1)}\left(p_{t}^{(1)}\right)=K-\alpha \log _{10}\left|p_{i}-p_{t}^{(1)}\right|, \quad i=1, \ldots, M,
$$




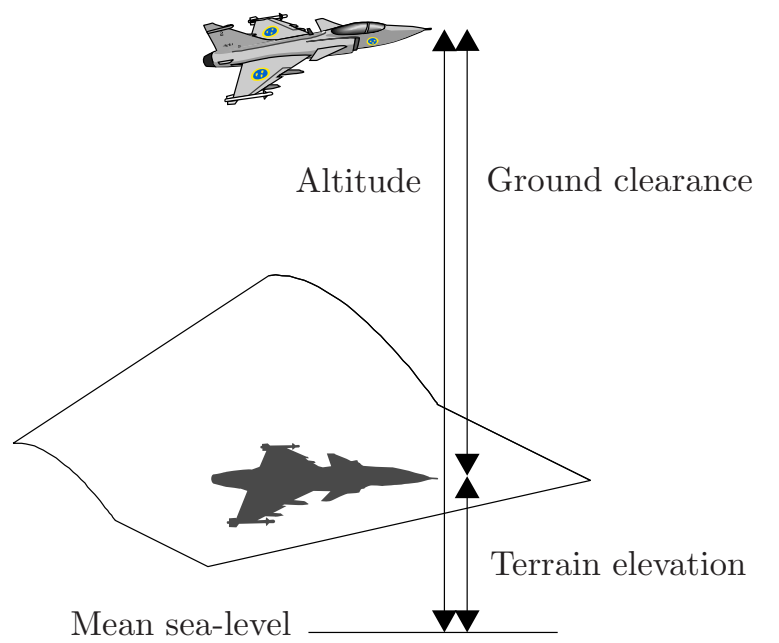

Fig. 1. Aircraft measures absolute altitude and height over ground from which terrain height $y$ is computed.

where $K=\log _{10} K_{1}[26]$. Analogously, we can consider the situation when we focus on the power or intensity transmitted or reflected from an object and received at one's own position. The measurement is thus modeled by

$$
h_{d}^{(2)}\left(p_{t}^{(1)}, p_{t}^{(2)}\right)=K-\alpha \log _{10}\left|p_{t}^{(1)}-p_{t}^{(2)}\right| .
$$

\section{B.2 Measurements of Relative Angle}

Similarly, the sensors can measure the relative angle between two positions (e.g. radar, IR, sonar, ultrasound). Given points of known positions $p_{i}, i=1, \ldots, M$, the relative angle measurements can be described by

$$
h_{e, i}^{(1)}\left(p_{t}^{(1)}\right)=\text { angle }\left\{p_{i}, p_{t}^{(1)}\right\}, i=1, \ldots, M .
$$

When relating the angle of an object to one's own position, we have

$$
h_{f}^{(2)}\left(p_{t}^{(1)}, p_{t}^{(2)}\right)=\operatorname{angle}\left\{p_{t}^{(1)}, p_{t}^{(2)}\right\}
$$

\section{B.3 Measurements of Relative Velocity}

Some sensors (e.g. radar) typically measure the Doppler shift of signal frequencies to estimate the magnitude of the relative velocity. This is essentially only applicable when relating the velocity of an object to one's own velocity. The measurements are categorized by

$$
h_{g, i}^{(2)}\left(v_{t}^{(1)}, v_{t}^{(2)}\right)=\left|v_{t}^{(2)}-v_{t}^{(1)}\right| .
$$

\section{B.4 Map Related Measurements}

Fig. 1 illustrates how ground altitude is computed from radar measurements of height over ground and barometric measurements from which altitude is computed. The measured terrain height together with relative movement from the INS build up a height profile as illustrated in Fig. 2, and the task is to find the current position and orientation of this profile on the map.

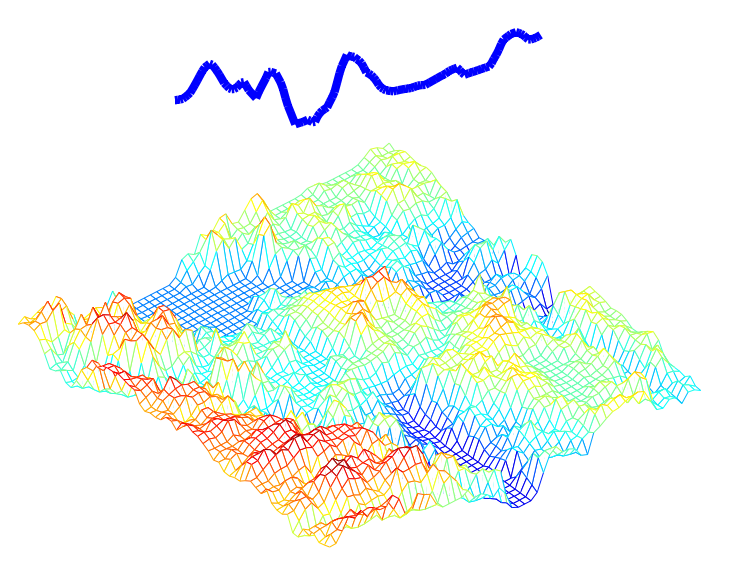

Fig. 2. Measured terrain elevation $y$ together with measured velocity can be seen as the profile above the terrain elevation map $h\left(p^{(1)}\right)$.

The measurement in terrain navigation is the measured ground height, and $h_{h}\left(p^{(1)}\right)$ is the height at point $p^{(1)}$ according to the Geographical Information System (GIS). Much effort has been spent on modeling the measurement error $e_{t}^{(1)}$ in a realistic way. It has turned out that a Gaussian mixture with two modes works well. One mode has zero mean, and the other a positive mean which corresponds to radar echos from the tree tops. The ground type in GIS can be used to switch the mean and variances in the Gaussian mixture. For instance, over sea there is only one mode with a small variance.

For map matching in the car positioning case, there is no real measurement. Instead, $h_{j}^{(1)}\left(p_{t}^{(1)}\right)$ denotes the distance to the nearest road, and the measurement

$$
y_{t}^{(1)}=h_{j}^{(1)}\left(p_{t}^{(1)}\right)+e_{t}^{(1)},
$$

should therefore be equal to zero. A simple and relevant noise model is white and zero mean Gaussian noise.

\section{Applications}

The applications discussed briefly in Section I are explored in further detail in the sequel. Typical state vectors, input signals and available (non-linear) sensor information are summarized in Table II. Motivations and more elaborative discussions regarding the applications and appropriate models are found in Sections IV, V, VI and VII.

\section{The Particle Filter}

\section{A. Recursive Bayesian Estimation}

Consider systems that are described by the generic state space model (1). The optimal Bayesian filter in this case is given below. For further details, consult [5].

Denote the set of available observations at time $t$ by

$$
Y_{t}=\left\{y_{0}, \ldots, y_{t}\right\}
$$

The Bayesian solution to compute the posterior distribution, $p\left(x_{t} \mid Y_{t}\right)$, of the state vector, given past observations, 


\begin{tabular}{|c|c|c|c|}
\hline Application & State vector & Input & Measurement equations \\
\hline Car positioning & $p_{t}^{(1)}$ & $v_{t}^{(1)}$ & $\begin{array}{l}\text { Road map } h_{j}\left(p_{t}^{(1)}\right) \text {, possibly GPS or base station } \\
\text { distances } h_{a, i}^{(1)}\left(p_{t}^{(1)}\right) \text {, base station powers } h_{c, i}^{(1)}\left(p_{t}^{(1)}\right)\end{array}$ \\
\hline Aircraft positioning & $p_{t}^{(1)}$ & $a_{t}^{(1)}$ & $\begin{array}{l}\text { Altitude } \operatorname{map}_{j} h_{j}\left(p_{t}^{(1)}\right) \text {, GPS or other reference } \\
\text { beacons } h_{a, i}^{(1)}\left(p_{t}^{(1)}\right)\end{array}$ \\
\hline Navigation in aircraft & $p^{(1)}, v^{(1)}, \delta a_{t}^{(1)}$ & $a_{t}^{(1)}$ & $\begin{array}{l}\text { Altitude map } h_{j}\left(p_{t}^{(1)}\right) \text {, GPS or other reference } \\
\text { beacons } h_{a, i}^{(1)}\left(p_{t}^{(1)}\right)\end{array}$ \\
\hline Tracking & $p_{t}^{(2)}, v_{t}^{(2)}$ & & $\begin{array}{l}\text { distance } h_{b}^{(2)}\left(p_{t}^{(1)}, p_{t}^{(2)}\right) \text {, bearing } h_{f}^{(2)}\left(p_{t}^{(1)}, p_{t}^{(2)}\right) \text {, } \\
\text { Doppler } h_{g}^{(2)}\left(p_{t}^{(1)}, p_{t}^{(2)}\right) \text {, intensity } h_{d}^{(2)}\left(p_{t}^{(1)}, p_{t}^{(2)}\right)\end{array}$ \\
\hline $\begin{array}{l}\text { Navigation and tracking } \\
\text { in aircraft }\end{array}$ & $p_{t}^{(1)}, v_{t}^{(1)}, \delta a_{t}^{(1)}, p_{t}^{(2)}, v_{t}^{(2)}$ & $a_{t}^{(1)}$ & $\begin{array}{l}\text { Altitude map } h_{j}\left(p_{t}^{(1)}\right) \text {, GPS or other reference } \\
\text { beacons } h_{a, i}^{(1)}\left(p_{t}^{(1)}\right) \\
\text { distance } h_{b}^{(2)}\left(p_{t}^{(1)}, p_{t}^{(2)}\right) \text {, bearing } h_{f}^{(2)}\left(p_{t}^{(1)}, p_{t}^{(2)}\right) \\
\text { Doppler } h_{g}^{(2)}\left(p_{t}^{(1)}, p_{t}^{(2)}\right) \text {, intensity } h_{d}^{(2)}\left(p_{t}^{(1)}, p_{t}^{(2)}\right)\end{array}$ \\
\hline $\begin{array}{l}\text { Navigation and tracking } \\
\text { in cars }\end{array}$ & $p_{t}^{(1)}, v_{t}^{(1)}, \delta a_{t}^{(1)}, p_{t}^{(2)}, v_{t}^{(2)}$ & $a_{t}^{(1)}$ & $\begin{array}{l}\text { Road map } h_{j}\left(p_{t}^{(1)}\right) \text {, possibly GPS or base station } \\
\text { distances } h_{a, i}^{(1)}\left(p_{t}^{(1)}\right) \text {, base station powers } h_{c, i}^{(1)}\left(p_{t}^{(1)}\right) \\
\text { distance } h_{b}^{(2)}\left(p_{t}^{(1)}, p_{t}^{(2)}\right) \text {, bearing } h_{f}^{(2)}\left(p_{t}^{(1)}, p_{t}^{(2)}\right), \\
\text { Doppler } h_{g}^{(2)}\left(p_{t}^{(1)}, p_{t}^{(2)}\right) \text {, intensity } h_{d}^{(2)}\left(p_{t}^{(1)}, p_{t}^{(2)}\right)\end{array}$ \\
\hline
\end{tabular}

TABLE II

List of considered applications With the corresponding state Vector (CF. TAble I), input signal and sensor information.

is given by [5]

$$
\begin{aligned}
p\left(x_{t+1} \mid Y_{t}\right) & =\int p\left(x_{t+1} \mid x_{t}\right) p\left(x_{t} \mid Y_{t}\right) d x_{t} \\
& =\int p_{f_{t}}\left(B_{f}^{\dagger}\left(x_{t+1}-A x_{t}-B_{u} u_{t}\right)\right) p\left(x_{t} \mid Y_{t}\right) d x_{t} \\
p\left(x_{t} \mid Y_{t}\right) & =\frac{p\left(y_{t} \mid x_{t}\right) p\left(x_{t} \mid Y_{t-1}\right)}{p\left(y_{t} \mid Y_{t-1}\right)} \\
& =\frac{p_{e_{t}}\left(y_{t}-h\left(x_{t}\right)\right) p\left(x_{t} \mid Y_{t-1}\right)}{c_{t}} \\
\hat{x}_{t}^{\mathrm{MMS}} & =\int x_{t} p\left(x_{t} \mid Y_{t}\right) d x_{t} \\
P_{t}^{\mathrm{MMS}} & =\int\left(x_{t}-\hat{x}_{t}^{\mathrm{MMS}}\right)\left(x_{t}-\hat{x}_{t}^{\mathrm{MMS}}\right)^{T} p\left(x_{t} \mid Y_{t}\right) d x_{t},
\end{aligned}
$$

where $\dagger$ denotes the Moore-Penrose pseudo-inverse, $c_{t}$ a normalization constant, and $\hat{x}_{t}^{\mathrm{MMS}}$ the minimum mean square (MMS) estimate.

If the noise distributions are independent, white and zero mean Gaussian with $\mathrm{E}\left(e_{t} e_{t}^{T}\right)=R, \mathrm{E}\left(f_{t} f_{t}^{T}\right)=Q$ and the measurement equation is linear in the state, i.e. $h\left(x_{t}\right)=C x_{t}$, the optimal solution is given by the Kalman estimator [29]. Table III summarizes the time and measurement update equations for the Kalman estimator.

\section{B. Particle Filter Implementation}

A numerical approximation to (4) is given in the following algorithm.

Algorithm 1: Particle Filter
1. Initialization: Generate $x_{0}^{i} \sim p_{x_{0}}, i=1, \ldots, N$. Each sample of the state vector is referred to as a particle. 2. Measurement update: Update the weights by the likelihood (more generally, any importance function, see [13]):

$$
w_{t}^{i}=w_{t-1}^{i} p\left(y_{t} \mid x_{t}^{i}\right)=w_{t-1}^{i} p_{e_{t}}\left(y_{t}-h\left(x_{t}^{i}\right)\right), i=1,2, \ldots, N
$$

and normalize to $w_{t}^{i}:=w_{t}^{i} / \sum_{i} w_{t}^{i}$. As an approximation to (4c), take

$$
\hat{x}_{t} \approx \sum_{i=1}^{N} w_{t}^{i} x_{t}^{i}
$$

3. Re-sampling:

(a) Bayesian bootstrap. Take $N$ samples with replacement from the set $\left\{x_{t}^{i}\right\}_{i=1}^{N}$ where the probability to take sample $i$ is $w_{t}^{i}$. Let $w_{t}^{i}=1 / N$. This step is also called Sampling Importance Re-sampling (SIR).

(b) Importance sampling. Only res-ample as above when the effective number of samples is less than a threshold $N_{t h}$

$$
N_{\mathrm{eff}}=\frac{1}{\sum_{i}\left(w_{t}^{i}\right)^{2}}<N_{t h}
$$

see [5], [14], [34], [35]. Here $1 \leq N_{\text {eff }} \leq N$, where the upper bound is attained when all particles have the same weight, and the lower bound when all probability mass is at one particle. The threshold can be chosen as $N_{t h}=2 N / 3$.

4. Prediction: Take a $f_{t}^{i} \sim p_{f_{t}}$ and simulate

$$
x_{t+1}^{i}=A x_{t}^{i}+B_{u} u_{t}+B_{f} f_{t}^{i}, i=1,2, \ldots, N .
$$

5. Let $t:=t+1$ and iterate to item 2 . 
The key point with re-sampling is to prevent high concentration of probability mass at a few particles. Without this step, some $w_{t}^{i}$ will converge to 1 and the filter would brake down to a pure simulation. The re-sampling can be efficiently implemented using a classical algorithm for sampling $N$ ordered independent identically distributed variables [5], [39].

It can be shown analytically [11], that under some conditions the estimation error is bounded by $g_{t} / N$. The function $g_{t}$ grows with time, but does not depend on the dimension of the state space. That is, in theory we can expect the same good performance for high order state vectors. This is one of the key reasons for the success of the particle filter compared to other numerical approaches such as the point mass filter (a numerical integration technique which can be seen as a deterministic particle filter) [5] and filter banks [24]. The computational steps are compared to the Kalman filter in Table III. Note that the most time consuming step in the Kalman filter is the Riccati recursion of the matrix $P$, which is not needed in the particle filter. The time update of the state is the same. Let $n_{x}$ denote the dimension of the state vector, and similar definitions for $n_{y}$ and $n_{f}$. As a first order approximation for large $n_{x}$, the Kalman filter is $\mathcal{O}\left(2 n_{x}^{3}\right)$ from the matrix times matrix multiplication $A P$, while the particle filter is $\mathcal{O}\left(N n_{x}^{2}\right)$ from the matrix times vector multiplication $A x$. This indicates that the particle filter is about 100 times slower in an application with $n_{x} \approx 5$ and $N \approx 10^{3}$. The difference becomes less when $n_{y}$ increases, in which case the measurement update becomes more complex. The non-linear function evaluation (preferably implemented as a table lookup) of $h(x)$ in the particle filter, has a counterpart of computing the gradient $C=d h(x) / d x$ in the Kalman filter, or any other linearization that is needed. In a multi-sensor application, the matrix inversion $\left(C P C^{T}+R\right)^{-1}$ may no longer be negligible. All in all, a precise comparison is hard to make, though it is worth pointing that the particle filter runs in real-time even in Matlab in several of the applications presented here.

\section{Sample Impoverishment}

When the particle filter is used in practice, we often wish to minimize the number of particles to reduce the computational burden. For many applications using recursive Monte Carlo methods, depletion or sample impoverishment may occur, i.e. the effective number of samples is reduced. This means that the particle cloud will not reflect the true density. Several different methods are proposed in the literature to reduce this problem.

By introducing an additional noise to the samples the depletion problem can be reduced. This technique is called jittering in [17], but a similar approach was introduced in [21] under the name roughening. In [15], the depletion problem is handled by introducing an additional Markov Chain Monte Carlo (MCMC) step to separate the samples

In [21], the so-called prior editing method is discussed. The estimation problem is delayed one time-step, so that the likelihood can be evaluated at the next time step. The idea is to reject particles with sufficiently small likelihood values, since they are not likely to be re-sampled. The update step is repeated until a feasible likelihood value is received. The roughening method could also be applied before the update step is invoked. The auxiliary particle filter [37] is constructed in such a way that we will simulate from particles associated with large predictive likelihoods directly. A two stage re-sampling may be used by this method.

\section{Rao-Blackwellization}

Despite the theoretical independence of accuracy on the particle dimension, it is well-known that the number of particles needs to be quite high for high-dimensional systems, see for instance Section VI for an illustration. To be able to use a small $N$, and also to reduce the risk of divergence, a procedure known as Rao-Blackwellization can be applied. The idea is to use the Kalman filter for the part of the state space model that is linear, and the particle filter for the other part. As a motivation, the state vector in inertial navigation can have as many as 27 states, and here the Kalman filter can be used for the 24 states while the particle filter applies on the three-dimensional position state. The extra work load is here minor.

The motion models given in Section II can actually be re-written in the form

$$
\begin{aligned}
\left(\begin{array}{c}
x_{t+1}^{\mathrm{pf}} \\
x_{t+1}^{\mathrm{kf}}
\end{array}\right) & =\left(\begin{array}{ll}
I & A^{\mathrm{pf}} \\
0 & A^{\mathrm{kf}}
\end{array}\right)\left(\begin{array}{c}
x_{t}^{\mathrm{pf}} \\
x_{t}^{\mathrm{kf}}
\end{array}\right)+\left(\begin{array}{c}
B_{u}^{\mathrm{pf}} \\
B_{u}^{\mathrm{kf}}
\end{array}\right) u_{t}+\left(\begin{array}{c}
B_{f}^{\mathrm{pf}} \\
B_{f}^{\mathrm{kf}}
\end{array}\right) f_{t} \\
y_{t} & =h\left(x_{t}^{\mathrm{pf}}\right)+e_{t},
\end{aligned}
$$

where $x_{t}^{\mathrm{pf}}$ (pf short for particle filter) and $x_{t}^{\mathrm{kf}}$ (kf short for Kalman filter) is a partition of the state vector with $f_{t}$ assumed Gaussian. The $e_{t}$ and $x_{0}^{\mathrm{pf}}$ can have arbitrarily given distributions. As the indices indicate, the Kalman filter will be applied to one part and the particle filter for the other part of the state vector.

For a derivation of the algorithm, see the Appendix or [36]. A similar result is presented in [12] for the general case, where the state space equation is linear and Gaussian, but one observes a $z_{t}$ instead of $y_{t}$, where the relation $p\left(z_{t} \mid y_{t}\right)$ is known. An algorithmically similar approach is given in [5], as an approximate solution to an altitude offset in terrain navigation. The result is a particle filter with $N$ particles estimating $x_{t}^{\mathrm{pf}}$. The difference to the standard particle filter algorithm (Algorithm 1) here is that the prediction step is done using

$$
\begin{aligned}
& x_{t+1}^{\mathrm{pf}, \mathrm{i}} \sim \mathrm{N}\left(x_{t}^{\mathrm{pf}, \mathrm{i}}+A^{\mathrm{pf}} \hat{x}_{t \mid t-1}^{\mathrm{kf}, \mathrm{i}}+B_{u}^{\mathrm{pf}} u_{t},\right. \\
&\left.A^{\mathrm{pf}} P_{t \mid t-1}^{\mathrm{kf}}\left(A^{\mathrm{pf}}\right)^{T}+B_{f}^{\mathrm{pf}} Q_{t}\left(B_{f}^{\mathrm{pf}}\right)^{T}\right) .
\end{aligned}
$$

Moreover, for each particle, one Kalman filter estimates 


\begin{tabular}{|l|l|l|}
\hline Algorithm & Kalman filter & Particle filter \\
\hline Time update & $x:=A x+B_{u} u$ & $f^{i} \sim p_{f}$ \\
& $P:=A P A^{T}+B_{f_{t}} Q B_{f_{t}}^{T}$ & $x^{i}:=A x^{i}+B_{u} u+B_{f} f^{i}$ \\
\hline Measurement update & $K=P C^{T}\left(C P C^{T}+R\right)^{-1}$ & $w^{i}:=w^{i} p_{e}\left(y-h\left(x^{i}\right)\right)$ \\
& $x:=x+K(y-C x)$ & \\
& $P:=P-K C P$ & \\
\hline
\end{tabular}

TABLE III

Comparison of KF and PF: Main Computational steps.

$$
\begin{aligned}
\left\{x_{t+1 \mid t}^{\mathrm{kf}, \mathrm{i}} ; i=1, . ., N\right\} \text { using } & \\
K_{t}= & P_{t \mid t-1}^{\mathrm{kf}}\left(A^{\mathrm{pf}}\right)^{T}\left(A^{\mathrm{pf}} P_{t \mid t-1}^{\mathrm{kf}}\left(A^{\mathrm{pf}}\right)^{T}+B_{f}^{\mathrm{pf}} Q_{t}\left(B_{f}^{\mathrm{pf}}\right)^{T}\right)^{-1} \\
\hat{x}_{t+1 \mid t}^{\mathrm{kf}, \mathrm{i}}= & \bar{A}^{\mathrm{kf}}\left(\hat{x}_{t \mid t-1}^{\mathrm{kf}, \mathrm{i}}+K_{t}\left(z_{t}^{i}-A^{\mathrm{pf}} \hat{x}_{t \mid t-1}^{\mathrm{kf}, \mathrm{i}}\right)\right)+ \\
& B_{u}^{\mathrm{kf}} u_{t}+B_{f}^{\mathrm{kf}}\left(B_{f}^{\mathrm{pf}}\right)^{\dagger} z_{t}^{i} \\
P_{t+1 \mid t}^{\mathrm{kf}}= & \bar{A}^{\mathrm{kf}}\left(P_{t \mid t-1}^{\mathrm{kf}}-K_{t} A^{\mathrm{pf}} P_{t \mid t-1}^{\mathrm{kf}}\right)\left(\bar{A}^{\mathrm{kf}}\right)^{T},
\end{aligned}
$$

where $\bar{A}^{\mathrm{kf}}=A^{\mathrm{kf}}-B_{f}^{\mathrm{kf}}\left(B_{f}^{\mathrm{pf}}\right)^{\dagger} A^{\mathrm{pf}}(\dagger$ denotes the MoorePenrose pseudo-inverse) and $z_{t}^{i}=\hat{x}_{t+1}^{\mathrm{pf}, \mathrm{i}}-\hat{x}_{t}^{\mathrm{pf}, \mathrm{i}}$.

Remark 1. The covariance $P_{t \mid t-1}^{\mathrm{kf}}$ and the Kalman gain $K_{t}$ are the same for all particles, implying a very efficient implementation of the $N$ parallel Kalman filters, where the $P$ and $K$ updates in Table III are done only once per time step.

Remark 2. The distribution for $x_{0}^{\mathrm{kf}}$ does not necessarily have to be Gaussian. We can approximate $p\left(x_{0}^{\mathrm{kf}}\right)$ arbitrarily well by $\left\{N\left(\hat{x}_{0 \mid-1}^{\mathrm{kf}, \mathrm{i}}, P_{0 \mid-1}^{\mathrm{kf}}\right) ; i=1, . ., N\right\}$.

Remark 3. The derivation still holds if an additional nonlinear term $g\left(x_{t}^{\mathrm{pf}}\right)$ enters the state dynamics for $x_{t}^{\mathrm{pf}}$.

Remark 4. The Kalman filter here applies to a state vector of dimension $n_{x}-n_{y}$, which is an improvement compared to dimension $\operatorname{dim} x$ as the derivation in [12] leads to. For large $n_{x}$, the reduction in complexity is approximately $\mathcal{O}\left(\frac{\left(n_{x}-n_{y}\right)^{3}}{n_{x}^{3}}\right)$.

The estimate of the particle filter part is computed in the normal way, and for the Kalman filter part we can take the MMS estimate (4c)

$$
\hat{x}_{t}^{\mathrm{kf}} \approx \sum_{i=1}^{N} w_{t}^{i} \hat{x}_{t \mid t-1}^{\mathrm{kf}, \mathrm{i}}
$$

with covariance $(4 \mathrm{~d})$

$$
P_{t}^{\mathrm{kf}} \approx P_{t \mid t-1}^{\mathrm{kf}}+\sum_{i=1}^{N} w_{t}^{i}\left(\hat{x}_{t \mid t-1}^{\mathrm{kf}, \mathrm{i}}-\hat{x}_{t \mid t-1}^{\mathrm{kf}}\right)\left(\hat{x}_{t \mid t-1}^{\mathrm{kf}, \mathrm{i}}-\hat{x}_{t \mid t-1}^{\mathrm{kf}}\right)^{T} .
$$

\section{Car Positioning}

Wheel speed sensors in ABS are available as standard components in the test car (Volvo V40). From this, yaw rate and speed information are computed as described in $[22]$. Therefore, the velocity vector $v_{t}^{(1)}$ is considered available as an input signal, and the motion model in (2a) with measurement equation given by (3a) is thus appropriate.
The initial position is either marked by the driver or given from a different system, e.g. a terrestrial wireless communications system, where crude position information is available today [16], or GPS. The initial area should cover an area not extending more than a couple of kilometers to limit the number of particles to a realizable number. With infinite memory and computation time, no initialization would be necessary.

The car positioning with map matching has been implemented in a car and the particle filter runs in real-time with sampling frequency $2 \mathrm{~Hz}$ on a modern laptop with a commercial digital road map. This corresponds to a measurement equation specified by $h_{j}^{(1)}\left(p_{t}^{(1)}\right)$ in Section II-B.4.

Fig. 3 shows a sequence of images of the particle cloud on a flight image of the local area. The driven path consists of a number of 90 degrees turns. Initially, the particles are spread uniformly over all admissible positions, that is, on the roads, covering an area of about one square kilometer. After the first turns, a few clouds are left. After 4-5 turns, the filter essentially has converged. One can note that the state evolution on the straight path extends the cloud along the road to take into account unprecise velocity information. Details of the implementation are found in [23], [25], while some comments on the divergence problem are given in the conclusions.

GPS is used as a reference positioning system. It provides reliable position estimates in rural areas, but is hampered in non line-of-sight situations and when the signals are attenuated by foliage etc. After convergence, the map matching particle filter is seen equal to, or even slightly better than, GPS in terms of performance, see Fig. 4. However, in test drives along forests, close to high buildings and tunnels, the GPS performance deteriorates quickly. Furthermore, the GPS has a convergence time of about 45 seconds when turned on, not shown in Fig. 4.

For comparison, the particle filter using map matching and filters based on measurements from a fictive terrestrial wireless communications system are applied to data from a simulation setup mimicking the real case above. The area is essentially covered by one macro cell, but yet another base station is assumed within measurable distance.

The base stations in a terrestrial wireless communications system act as beacons by transmitting pilot signals of known power. The mobile station monitors the $M$ (in GSM (Global System for Mobile Communications), $M=5$ ) strongest signals, and reports regularly (or event-driven) 

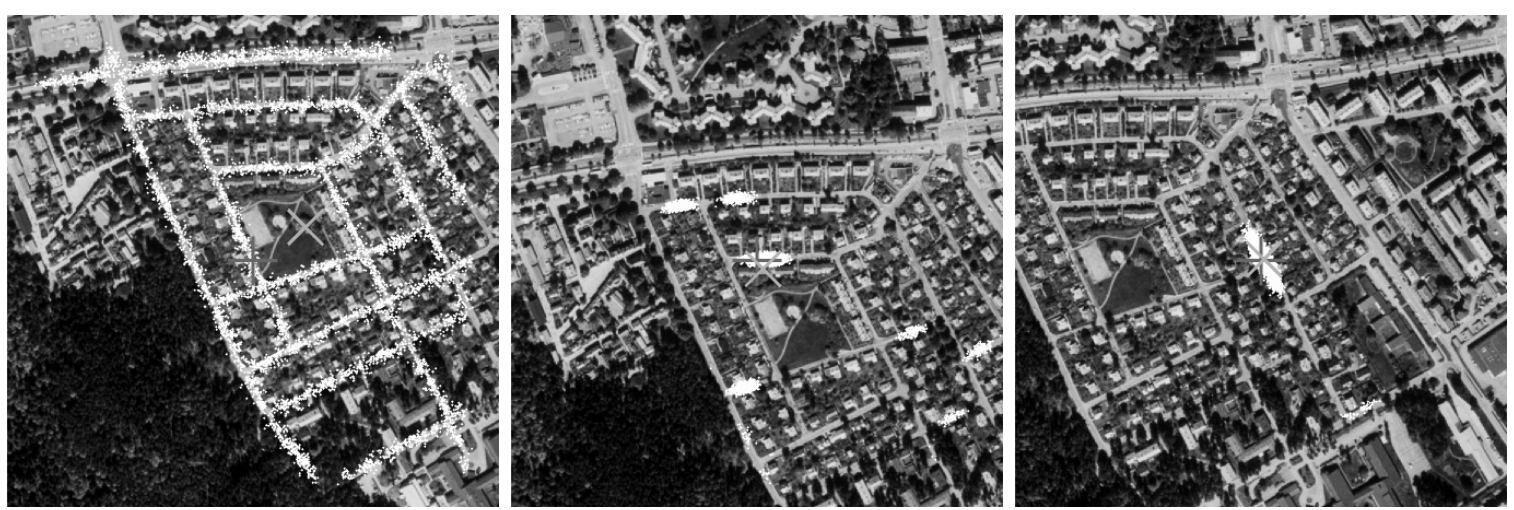

Fig. 3. Car positioning: Sequence of illustrations of particle clouds (white dots) plotted on a flight image for visualization. The center point 't' shows the true position and ' $\mathrm{x}$ ' the estimate.

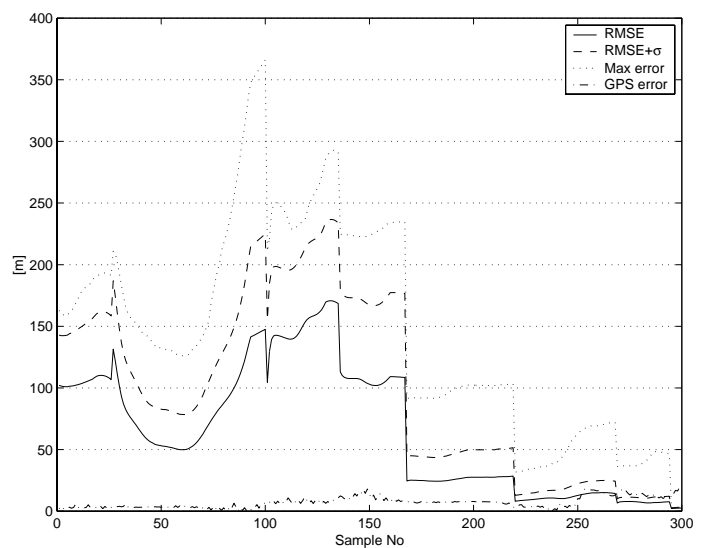

Fig. 4. Car positioning: RMSE for particle filter and GPS, respectively.

the list to the network. Based on these lists, the network centrally transfers connections from one base station to another (hand-over) when the mobile is moving during the service session. According to the empirical model by Okumura-Hata [26], this provides $M$ measurement equations as in (3e), one for each available base station (in this simulation, $M=2)$, and $p_{e}(e) \in \mathrm{N}\left(0, \sigma_{e}^{2}\right)$, where $\sigma_{e}=6$ dB. Similar measurements, but with a different motion model (the velocity is unknown) are used in [28]. Pointmass implementation of estimators based on RF measurements is also discussed in [9].

To provide more accurate positioning via RF measurements, future mobile stations will be able to estimate the traveled distance of radio signals from a multitude of base stations. In the ideal case, the signals have traveled without reflections to the mobile station (line-of-sight situation), and the estimates describe the distance to the base stations. The $M$ ( $M$ is typically 1-3) measurement equations can thus be modeled by (3c), and they represent a rather ideal situation. Moreover, the noise is modeled as $p_{e}(e) \in \mathrm{N}\left(0, \sigma_{e}^{2}\right)$, where $\sigma_{e}=3 \mathrm{~dB}$. The received power measurements discussed above are available today, but are of worse accuracy due to unmodeled power variations.

A third alternative is to simply integrate the relative movements provided by the ABS (dead-reckoning). MonteCarlo simulations based on these different approaches are summarized in Figure 5. It is interesting to note that map matching provides a position accuracy of roughly the same accuracy as with accurate distance measurements (which would almost never be the case in a real situation), without relying on external signals. Furthermore, integrating the ABS signals directly yields an increasing error over time.

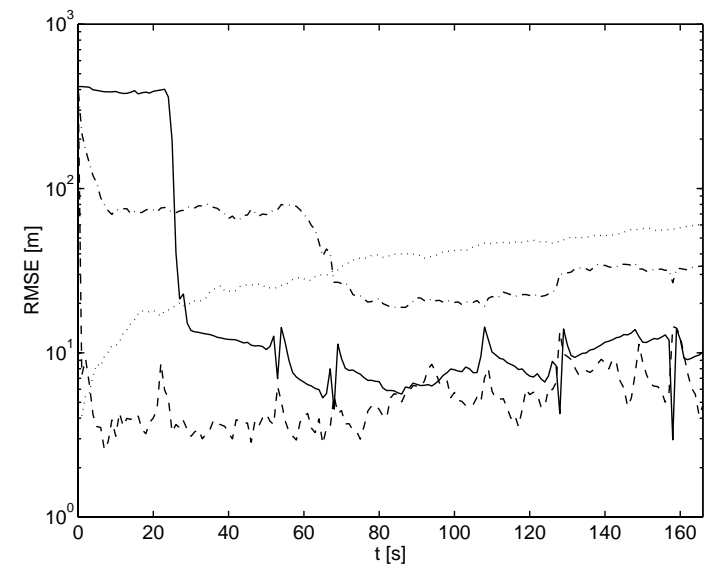

Fig. 5. RF positioning: Monte-Carlo performance over time in the simulated scenario. The map matching (solid) needs some 25 seconds to converge, but after this burn-in time, the algorithm provides $\mathrm{RMSE}=8.7 \mathrm{~m}$. This is almost as good as with ideal distance measurements to two base stations (dashed) with $\mathrm{RMSE}=7.0 \mathrm{~m}$. For comparison, power measurements (dash-dotted) yield RMSE $=36 \mathrm{~m}$, and dead-reckoning (dotted) a steadily increasing error with $\mathrm{RMSE}=50 \mathrm{~m}$.

\section{Terrain Elevation Matching}

The air fighter JAS 39 Gripen is equipped with an accurate radar altimeter and a digital map. This corresponds to the measurement equation characterized by $h_{h}\left(p^{(1)}\right)$ in Section II-B.4. The velocity vector is obtained by integrating the acceleration provided by the inertial navigation system. Since $v_{t}^{(1)}$ is available as an input signal, the motion model in $(2 \mathrm{a})$ is appropriate.

The particle filter has been applied to a number of flight tests on the fighter JAS 39 Gripen, and Fig. 6 shows the 


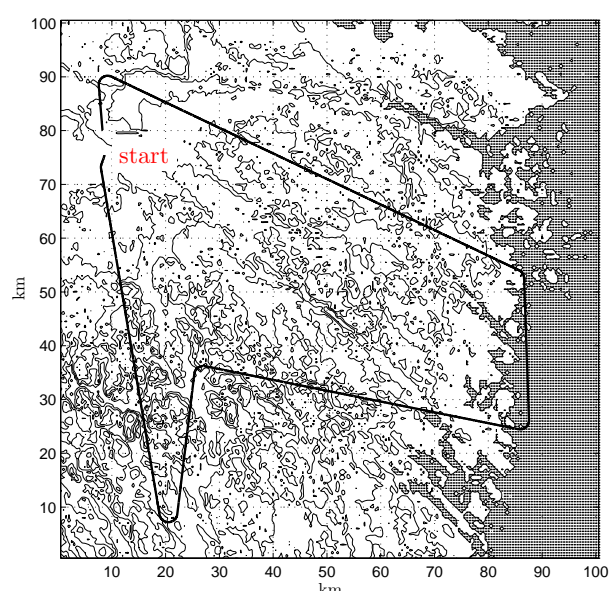

Fig. 6. Terrain navigation: Test track over a part of south-eastern Sweden.

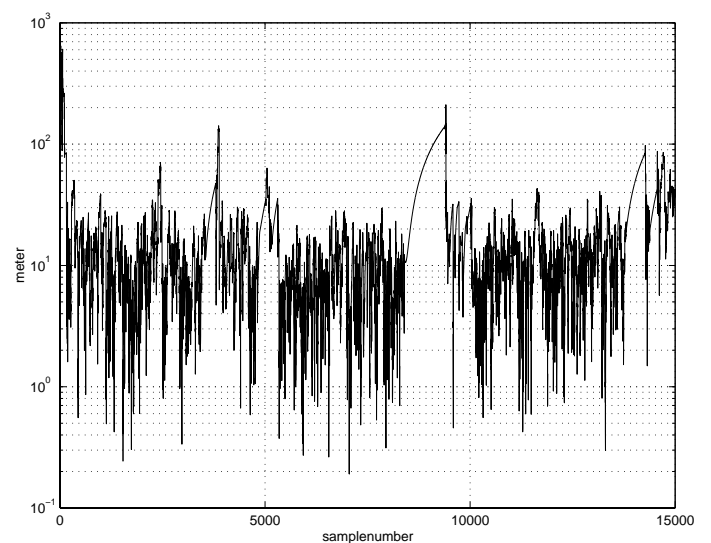

Fig. 7. Terrain navigation: Estimation error relative a GPS reference, as a function of sample number. Note the growth in error over open water.

path in one of them. In these tests, differential GPS (DGPS) is taken as the true position, and the resulting position error is shown in Fig. 7. The accuracy beats the first generation system, and comes down to the value of the point mass filter described in [8]. Since the point mass filter satisfies the Cramer-Rao lower bound, see [6], there is no better filter. The advantage of the particle filter over the point mass filter is firstly a much less complex algorithm occupying only some 30 lines of code (Ada), and secondly the possibility to extend the functionality by including other parameters such as barometric height offset in the state vector (that is, increasing the particle dimension). Saab has evaluated the deterministic particle filter in Gripen in parallel with the first generation system with superior results, while the particle filter described here-in so far is run off-line.

\section{INTEGRATED NAVIGATION SYSTEMS}

As a simplified study to illustrate the Rao-Blackwellization procedure, a two-dimensional navigation model with six states is used according to (2b) and the measurement of
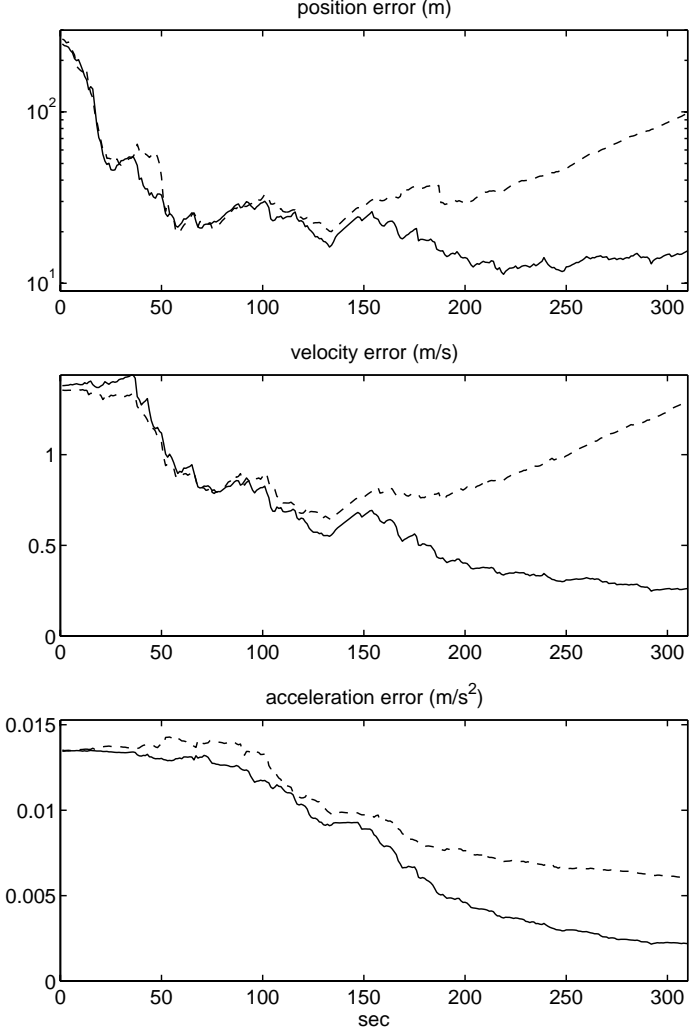

Fig. 8. RMSE based on 100 Monte Carlo simulations for the particle filter using 60000 particles (dashed lines) and the RaoBlackwellized filter using 4000 particles (solid lines).

position is taken from the terrain navigation algorithm according to Section II-B.4. It should be noted that the twodimensional navigation model is valid only when the earth is modeled as flat. As soon as one accounts for the curvature of the earth the model becomes more complicated, see [10]. In practice there also exists gyro sensor errors which further complicate the problem.

In Fig. 8, the result is shown for the particle filter when using $N=60000$ particles (dashed lines). The performance is pretty bad, and it quickly deteriorates even more when the number of particles is decreased. In particular the transient requires many particles. The basic problem is high dimensionality and small process noise. On the other hand, following the Rao-Blackwellization procedure we partition the state vector and rewrite the motion model according to $(5)$, with

$$
x_{t}^{\mathrm{pf}}=p_{t}^{(1)}, \quad x_{t}^{\mathrm{kf}}=\left(\begin{array}{c}
v_{t}^{(1)} \\
\delta a_{t}^{(1)}
\end{array}\right) .
$$

The result from applying this Rao-Blackwellized filter using only $N=4000$ particles is also shown in Fig. 8 (solid lines), and the performance enhancement is significant.

\section{TARget TRACKING}

The standard approach to target tracking is based on (extended) Kalman filters [3], [42]. Bearings-only target 
tracking was introduced as the illustration of particle filters in [21]. Since then, bearings-only target tracking has been used in many investigations, see for instance several of the chapters in [13]. A more realistic scenario is investigated in [31]. Here, the case of radar measurements where also range is available is discussed, which occurs in different applications, such as air traffic control (ATC) and collision avoidance. Often linear models such as (1) can be used, but nonlinear state equations are also used. For instance, when the tracking object is moving in straight paths or on circular segments, different variations of the so-called coordinated turn model [4] can be utilized. For maneuvering targets, multiple models are used to enhance tracking performance. The Interacting Multiple Model (IMM) [4] is one classical multiple model algorithm based on the interaction of several extended Kalman filters [1]. Hard constraints on system states, such as velocity and acceleration boundaries or obstacles from the terrain may introduce nonlinearities in many applications, which could degrade performance if not handled by the tracking filter. Two different applications will be presented in more detail below. It is here important to note that realistic measurements (3g) can easily be used, modeling the radar loob in the angle noise distribution, and (3c), with uniform range noise distribution.

An important aspect of target tracking is association [3], [42]; to which object should a certain measurement be associated? This is a discrete problem, and attempts to include this in a particle filter framework are described in [2], [41], [20], [27], [18], [38], [7], [32].

\section{A. Air Traffic Control (ATC)}

In [30], a simple nearly coordinated turn model [4] was used for an ATC radar application. In the simulation study presented in Table VII-A, two different simulation based methods are compared to the state-of-the-art IMM method. The particle filter algorithms tested are the Bayesian bootstrap method (3a) and APF [37]. The particle filters are here extended to the multiple model case, where target maneuvers are according to a Markov chain. Three different turn assumptions were made (right/left turn or straight flying) in the simulations presented. The true path projected in the horizontal plane is viewed in Figure 9. It was generated with a true turn rate value chosen as an intermediate value of the turn rate used in the multiple model conditioning, thus allowing the IMM to mix between models, and the particle filter process noise to perform the turn interaction. The incorporation of hard constraints on the velocity is also straightforward for the particle filter case. The radar sensor used in the application measures range, azimuth and elevation at a rather low update rate, to emulate a track-while-scan (TWS) behavior. In Table VII-A the IMM method is compared to the particle filters and measurements only, viewing the position RMSE for 100 Monte Carlo simulations. For the Bayesian bootstrap case, two simulations diverged. Depending on the choice of process noise, the slight difference between the IMM and the Bayesian bootstrap may change. The marginalized density is also shown in Figure 10 together

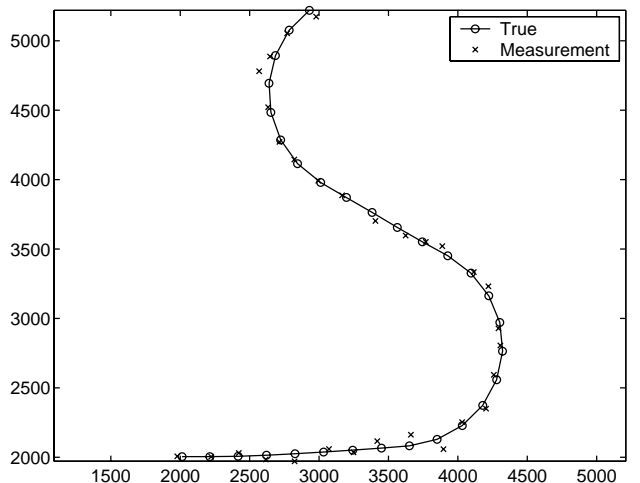

Fig. 9. Target tracking: RMSE from 100 Monte Carlo simulations, 800 particles
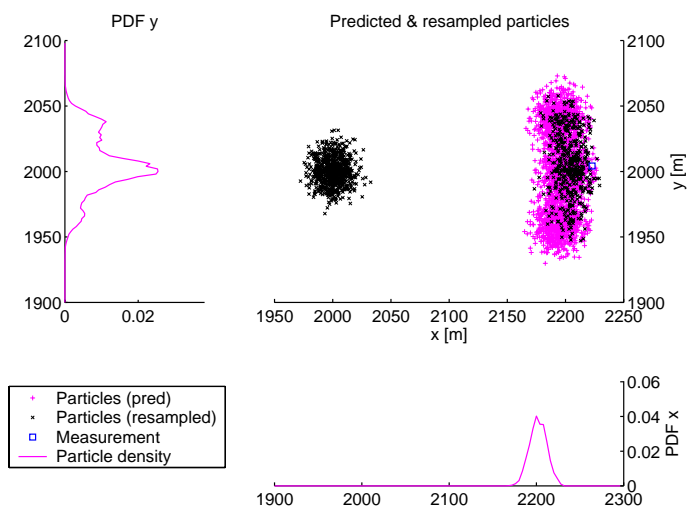

Fig. 10. Target tracking: Particle cloud and density

with the particle cloud.

\section{B. Collision Avoidance}

The coordinated turn model can be used for collision avoidance to track the car position and predict future positions. The goal of the prediction in this case is not necessarily to get an as good point estimate as possible. Instead, we are interested in the whole distribution of possible maneuvers. Figure 11 shows a simulation where the collision is still avoidable. This would not be obvious from just looking at the point estimate.

The main contributions to the process noise come from the driver's action via steering wheel, gas and brake. A lot of effort has to be spent on how to choose the process noise so that it corresponds to the driver's behavior and the physical limitations of the car. The vehicle and driver behaviors change significantly for different speeds of the

\begin{tabular}{|l|l|l|l|l|}
\hline & APF & Bootstrap & IMM-3 & Measurements \\
\hline RMSE & 34.03 & 40.84 & 42.20 & 63.96 \\
\hline
\end{tabular}

\section{TABLE IV}

TARget tracking: RMSE COMPARison for ATC Monte CARlo SimUlations. 


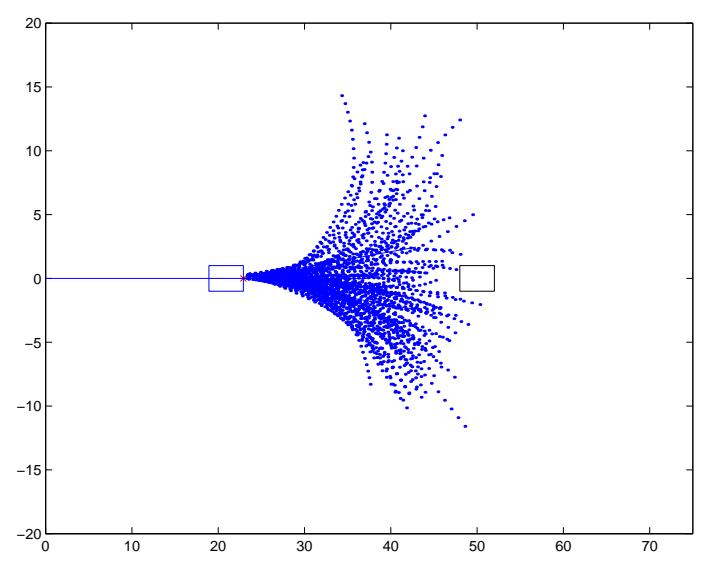

Fig. 11. Collision avoidance: The left rectangle is the own car, which is approaching rapidly the right rectangle. The trajectories indicate 31-step ahead prediction using 100 particles. There are still possible trajectories avoiding collision, of which the driver will most probably choose one. Thus, no active control is needed at this stage.

vehicle. Thus, in order to get a good prediction with this model, it is necessary to let the process noise $f_{t}$ change with different speeds. It is also important in this application to incorporate knowledge about the environment to improve the prediction. For example, it is likely that the car will travel on the road and if there are some hard boundaries like rails or other stationary objects these are hard constraints on the car's movement.

\section{Conclusions And Discussion}

We have given a general framework for positioning and navigation applications based on a flexible state space model and a particle filter. Five applications illustrate its use in practice. Evaluations in real-time, off-line on real data and in simulation environments show a clear improvement in performance compared to existing Kalman filter based solutions, where the new challenge is to find nonlinear relations, state constraints and non-Gaussian sensor models that provide the most information about the position. Thus, modeling is the most essential step in this approach, compared to the various implementations of the Kalman filter found in this context (linearization issues, choice of state co-ordinates, filter banks, Gaussian sum filters, etc.).

General conclusions from the implementations are as follows: A choice of state coordinates making the state equation linear is beneficial for computation time and opens up the possibility for Rao-Blackwellization. This procedure enables a significant decrease in the particle state dimension. The evaluation of the likelihood one step ahead before re-sampling (APF, prior editing) is, together with adding extra state noise (jittering, roughening), crucial for avoiding divergence, and implies that the number of particles can be decreased further. Our implementations run in real-time $(2 \mathrm{~Hz})$, even in Matlab, and have some 2000 particles.

Open questions for further research and development are listed below:

- Divergence tests. It is essential to have a reliable way to detect divergence and to restart the filter (for the latter, see the transient below). For car positioning, the number of resamplings in the prior editing step turned out to be a very good indicator of divergence. Another idea, used in the terrain navigation implementation where the sampling rate is higher than necessary, is to split up the measurements to a filter bank, so that particle filter number $i, i=1,2, \ldots, n$ gets every $n$ 'th sample. The result of these $n$ particle filters are approximately independent and voting can be used to restart each filter. This has turned out to be an efficient way to remove the outliers in data.

- Transient improvement. The time it takes until the estimate accuracy comes down to the stationary value (the Cramer-Rao bound) depends on the number of particles. Given limited computational time, it may be advantageous to increase the number of particles $N$ after a restart and discard samples in such a way that $N / T_{s}$ is constant.

- Since the particle filter has shown good improvement over linearization approaches, it is tempting to try even more accurate non-linear models. In particular, the flight dynamics of one's own vehicle is known and indeed used in modelbased control, but is very rare in navigation applications, see [33] for one attempt in this direction. In that study, it seems that the computational burden and linearization errors imply no gain in total performance. As a possible improvement, the particle filter may take full advantage of a more accurate model, where parts of the non-linear dynamics from driver/pilot inputs are incorporated.

\section{ACKNOWLEDGMENT}

The competence center ISIS at Linköping University has brought all of the authors together and provides funding for Rickard and Per-Johan. We are very grateful to Christophe Andrieu and Arnoud Doucet for our fruitful discussions on the theoretical subjects. We want to acknowledge our gratitude to the master students Magnus Ahlström, Marcus Calais, who have implemented the terrain navigation filter, and Peter Hall, who implemented the car positioning filter, and the supporting companies SAAB Dynamics and NIRA Dynamics, respectively.

\section{APPENDIX}

For the derivation of the Rao-Blackwellized algorithm given in Section III-D, suppose first that the particle filter part of the state vector is known. That is, the sequence $X_{t}^{\mathrm{pf}}=\left\{x_{0}^{\mathrm{pf}}, \ldots, x_{t}^{\mathrm{pf}}\right\}$ is known. We can, temporally, consider $z_{t}=x_{t+1}^{\mathrm{pf}}-x_{t}^{\mathrm{pf}}$ as the measurement. The state space model is here

$$
\begin{aligned}
x_{t+1}^{\mathrm{kf}} & =A^{\mathrm{kf}} x_{t}^{\mathrm{kf}}+B_{u}^{\mathrm{kf}} u_{t}+B_{f}^{\mathrm{kf}} f_{t} \\
z_{t} & =A^{\mathrm{pf}} x_{t}^{\mathrm{kf}}+B_{u}^{\mathrm{pf}} u_{t}+B_{f}^{\mathrm{pf}} f_{t} .
\end{aligned}
$$

Since this model is linear and Gaussian, the optimal solution is provided by the Kalman filter. We then know that $p\left(x_{t}^{\mathrm{kf}} \mid X_{t}^{\mathrm{pf}}\right)$ is Gaussian, so

$$
x_{t}^{\mathrm{kf}}\left|X_{t}^{\mathrm{pf}}=x_{t}^{\mathrm{kf}}\right| Z_{t-1} \sim N\left(\hat{x}_{t \mid t-1}^{\mathrm{kf}}, P_{t \mid t-1}^{\mathrm{kf}}\right),
$$


where $\hat{x}_{t \mid t-1}^{k f}$ and $P_{t \mid t-1}^{k f}$ are given by the Kalman filter equations adjusted for correlated noise [24],

$$
\begin{aligned}
K_{t}= & P_{t \mid t-1}^{\mathrm{kf}}\left(A^{\mathrm{pf}}\right)^{T}\left(A^{\mathrm{pf}} P_{t \mid t-1}^{\mathrm{kf}}\left(A^{\mathrm{pf}}\right)^{T}+B_{f}^{\mathrm{pf}} Q_{t}\left(B_{f}^{\mathrm{pf}}\right)^{T}\right)^{-1} \\
\hat{x}_{t+1 \mid t}^{\mathrm{kf}}= & \bar{A}^{\mathrm{kf}}\left(\hat{x}_{t \mid t-1}^{\mathrm{kf}}+K_{t}\left(z_{t}-A^{\mathrm{pf}} \hat{x}_{t \mid t-1}^{\mathrm{kf}}\right)\right)+ \\
& B_{u}^{\mathrm{kf}} u_{t}+B_{f}^{\mathrm{kf}}\left(B_{f}^{\mathrm{pf}}\right)^{\dagger} z_{t} \\
P_{t+1 \mid t}^{\mathrm{kf}}= & \bar{A}^{\mathrm{kf}}\left(P_{t \mid t-1}^{\mathrm{kf}}-K_{t} A^{\mathrm{pf}} P_{t \mid t-1}^{\mathrm{kf}}\right)\left(\bar{A}^{\mathrm{kf}}\right)^{T},
\end{aligned}
$$

where $\bar{A}^{\mathrm{kf}}=A^{\mathrm{kf}}-B_{f}^{\mathrm{kf}}\left(B_{f}^{\mathrm{pf}}\right)^{\dagger} A^{\mathrm{pf}}(\dagger$ denotes the MoorePenrose pseudo-inverse).

Now, to compute $p\left(x_{t} \mid Y_{t}\right)=p\left(x_{t}^{\mathrm{pf}}, x_{t}^{\mathrm{kf}} \mid Y_{t}\right)$, note that

$$
p\left(X_{t}^{\mathrm{pf}}, x_{t}^{\mathrm{kf}} \mid Y_{t}\right)=p\left(x_{t}^{\mathrm{kf}} \mid X_{t}^{\mathrm{pf}}\right) p\left(X_{t}^{\mathrm{pf}} \mid Y_{t}\right)
$$

We only have to compute $p\left(X_{t}^{\mathrm{pf}} \mid Y_{t}\right)$. Repeated use of Bayes' rule gives

$$
p\left(X_{t}^{\mathrm{pf}} \mid Y_{t}\right)=\frac{p\left(y_{t} \mid x_{t}^{\mathrm{pf}}\right) p\left(x_{t}^{\mathrm{pf}} \mid X_{t-1}^{\mathrm{pf}}\right)}{p\left(y_{t} \mid Y_{t-1}\right)} p\left(X_{t-1}^{\mathrm{pf}} \mid Y_{t-1}\right) .
$$

We have a nonlinear and non-Gaussian measurement equation, so to solve the measurement update, the particle filter will be used to approximate this distribution. The particle predictions $p\left(x_{t+1}^{\mathrm{pf}} \mid X_{t}^{\mathrm{pf}}\right)$ are given by

$$
x_{t+1}^{\mathrm{pf}}\left|X_{t}^{\mathrm{pf}}=x_{t}^{\mathrm{pf}}+A^{\mathrm{pf}} x_{t}^{\mathrm{kf}}\right| X_{t}^{\mathrm{pf}}+B_{u}^{\mathrm{pf}} u_{t}+B_{f}^{\mathrm{pf}} f_{t},
$$

so $p\left(x_{t+1}^{\mathrm{pf}} \mid X_{t}^{\mathrm{pf}}\right)$ is given by

$$
\begin{aligned}
& N\left(x_{t}^{\mathrm{pf}}+A^{\mathrm{pf}} \hat{x}_{t \mid t-1}^{\mathrm{kf}}+B_{u}^{\mathrm{pf}} u_{t},\right. \\
& \left.\quad A^{\mathrm{pf}} P_{t \mid t-1}^{\mathrm{kf}}\left(A^{\mathrm{pf}}\right)^{T}+B_{f}^{\mathrm{pf}} Q_{t}\left(B_{f}^{\mathrm{pf}}\right)^{T}\right) .
\end{aligned}
$$

Finally, note that the derivation does not change if we use the fictitious measurement $z_{t}=x_{t+1}^{\mathrm{pf}}-g\left(x_{t}^{\mathrm{pf}}\right)$ for an arbitrary non-linear function, which is Remark 3.

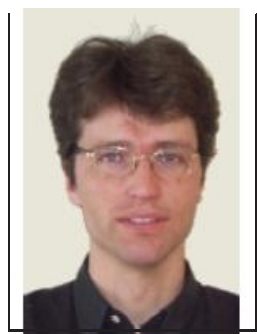

Fredrik Gustafsson is professor in Communication Systems at the Department of Electrical Engineering at Linköping University. He received the M.S. degree in electrical engineering in 1988 and the Ph.D. degree in automatic control in 1992, both from Linköping University, Sweden. His research is focused on statistical methods in signal processing, with applications to automotive, avionic and communication systems. He is an associate editor of IEEE Transactions of Signal Processing.

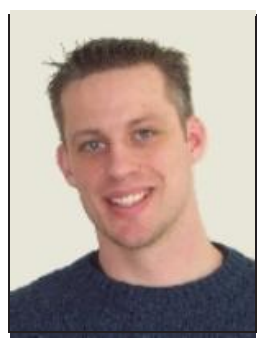

Fredrik Gunnarsson is a research associate at Communications Systems, Department of Electrical Engineering, Linköping University. He received the M.Sc. degree in applied physics and electrical engineering in 1996 and the $\mathrm{Ph} . \mathrm{D}$. degree in electrical engineering in 2000, both from Linköping University, Sweden. His research interests include control and signal processing in terrestrial wireless communications systems and automotive applications.

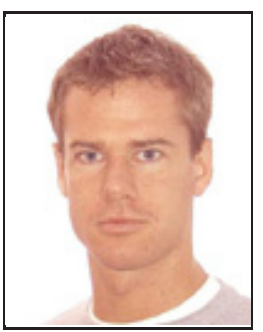

Niclas Bergman is with SaabTech Systems. He received his M.Sc. degree in applied physics and electrical engineering in 1995 and the Ph.D. degree in electrical engineering in 1999, both from Linköping University, Sweden. He is currently working with research and development in the areas of target tracking and navigation, and responsible for the coordination of data fusion activities within the Saab group.

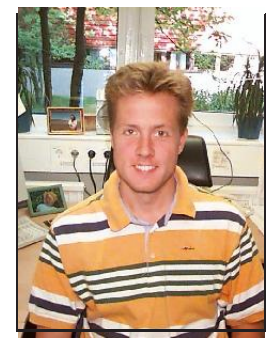

Urban Forssell Urban Forssell is president and CEO of NIRA Dynamics AB. The company focuses on advanced signal processing and control in vehicles. He received his M.Sc. degree in applied physics and electrical engineering in 1995 and the Ph.D. degree in automatic control in 1999, both from Linköping University, Sweden.

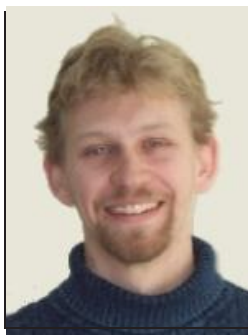

Jonas Jansson is employed at Volvo Car Corporation with developing a collision avoidance system. He is since 1999 spending $50 \%$ of his time as a PhD student at Linköping University. His current research interests focus on particle filter implementation of navigation and tracking systems.

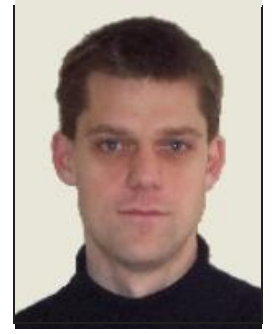

Rickard Karlsson has worked at SAAB Dynamics with target tracking and sensor fusion since 1997. He is since 1999 spending half his time as a PhD student at Linköping University. His current research interests focus on particle filter implementation of target tracking algorithms with radar and/or IR sensors. 


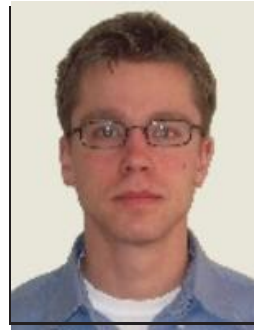

Per-Johan Nordlund has worked at SAAB Gripen with developing a new version of the navigation system for the fighter JAS 39 Gripen for several years. He is since 1999 spending $75 \%$ of his time as a $\mathrm{PhD}$ student at Linköping University. His current research interests focus around particle filter implementation of integrated navigation systems with particular attention to complexity aspects and fault detection.

\section{REFERENCES}

[1] B.D.O. Anderson and J.B. Moore. Optimal filtering. Prentice Hall, Englewood Cliffs, NJ., 1979.

[2] D. Avitzour. Stochastic simulation Bayesian approach to multitarget tracking. IEE Proc. on Radar, Sonar and Navigation, 142(2), 1995.

[3] Y. Bar-Shalom and T. Fortmann. Tracking and Data Association, volume 179 of Mathematics in Science and Engineering. Academic Press, 1988.

[4] Y. Bar-Shalom and X.R. Li. Estimation and tracking: principles, techniques, and software. Artech House, 1993.

[5] N. Bergman. Recursive Bayesian Estimation: Navigation and Tracking Applications. Dissertation nr. 579, Linköping University, Sweden, 1999.

[6] N. Bergman. Posterior Cramér-Rao bounds for sequential estimation. In A. Doucet, N. de Freitas, and N. Gordon, editors, Sequential Monte Carlo Methods in Practice. Springer-Verlag, 2001.

[7] N. Bergman and A. Doucet. Markov Chain Monte Carlo data association for target tracking. In IEEE Conference on Acoustics, Speech and Signal Processing, 2000.

[8] N. Bergman, L. Ljung, and F. Gustafsson. Terrain navigation using Bayesian statistics. IEEE Control System Magazine, 19(3):33-40, 1999.

[9] J. Blom, F. Gunnarsson, and F. Gustafsson. Estimation in cellular radio systems. In Proc. IEEE International Conference on Acoustics, Speech, and Signal Processing., Phoenix, AZ, USA., March 1999.

[10] K.R. Britting. Inertial Navigation Systems Analysis. Wiley Interscience, 1971.

[11] D. Crisan and A. Doucet. Convergence of sequential Monte Carlo methods. Technical Report CUED/F-INFENG/TR381, Signal Processing Group, Department of Engineering, University of Cambridge, 2000.

[12] A. Doucet and C. Andrieu. Particle filtering for partially observed Gaussian state space models. Technical Report CUED/FINFENG/TR393, Department of Engineering, University of Cambridge CB2 1PZ Cambridge, September 2000.

[13] A. Doucet, N. de Freitas, and N. Gordon, editors. Sequential Monte Carlo Methods in Practice. Springer Verlag, 2001.

[14] A. Doucet, S.J. Godsill, and C. Andrieu. On sequential simulation-based methods for Bayesian filtering. Statistics and Computing, 10(3):197-208, 2000.

[15] A. Doucet, N.J. Gordon, and V. Krishnamurthy. Particle Filters for State Estimation of Jump Markov Linear Systems. IEEE Trans. on Signal Processing, 49(3):613-624, March 2001.

[16] C. Drane, M. Macnaughtan, and C. Scott. Positioning GSM telephones. IEEE Communications Magazine, 36(4), 1998.

[17] P. Fearnhead. Sequential Monte Carlo methods in filter theory. $\mathrm{PhD}$ thesis, University of Oxford, 1998.

[18] S. Geman and D. Geman. Stochastic relaxation, Gibbs distributions and the Bayesian restoration of images. IEEE Trans. on Pattern Analysis and Machine Intelligence, 6:721-741, 1984.

[19] W. Gilks, S. Richardson, and D. Spiegelhalter. Markov Chain Monte Carlo in practice. Chapman \& Hall, 1996.

[20] N.J. Gordon. A hybrid bootstrap filter for target tracking in clutter. In IEEE Transactions on Aerospace and Electronic Systems, volume 33, pages 353-358, 1997.

[21] N.J. Gordon, D.J. Salmond, and A.F.M. Smith. A novel approach to nonlinear/non-Gaussian Bayesian state estimation. In IEE Proceedings on Radar and Signal Processing, volume 140, pages 107-113, 1993.

[22] F. Gustafsson, S. Ahlqvist, U. Forssell, and N. Persson. Sensor fusion for accurate computation of yaw rate and absolute velocity. In SAE 2001, Detroit, 2001.
[23] F. Gustafsson, U. Forssell, and P. Hall. Car positioning system. Swedish patent application nr SE0004096-4, 2000.

24] Fredrik Gustafsson. Adaptive filtering and change detection. John Wiley \& Sons, Ltd, 2000.

[25] P. Hall. A Bayesian approach to map-aided vehicle positioning. Master Thesis LiTH-ISY-EX-3104, Dept of Elec. Eng. Linköping University, S-581 83 Linköping, Sweden, 2001. In Swedish.

[26] M. Hata. Empirical formula for propagation loss in land mobile radio services. IEEE Transactions on Vehicular Technology, $29(3), 1980$.

[27] C. Hue, J.P. Le Cadre, and P. Pérez. Tracking multiple objects with particle filtering. Technical report, Research report IRISA, No1361, Oct 2000.

[28] H. Jwa, S. Kim, X. Cho, and J. Chun. Position tracking of mobiles in a cellular radio network using the constrained bootstrap filter. In Proc. National Aerospace Electronics Conference, Dayton, OH, USA, October 2000.

[29] T. Kailath, A.H. Sayed, and B. Hassibi. Linear estimation. Information and System Sciences. Prentice-Hall, Upper Saddle River, New Jersey, 2000.

[30] R. Karlsson and N. Bergman. Auxiliary particle filters for tracking a maneuvering target. In IEEE Conference on Decision and Control, Sydney, Australia, Dec 2000.

[31] R. Karlsson and F. Gustafsson. Range estimation using angleonly target tracking with particle filters. In Proc. of the American Control Conference, Arlington, Virginia, U.S.A, June 2001.

[32] Rickard Karlsson and Fredrik Gustafsson. Monte Carlo data association for multiple target tracking. In IEE Target tracking: Algorithms and applications, The Netherlands, Oct 2001.

[33] M. Koifman and I.Y. Bar-Itzhack. Inertial navigation system aided by aircraft dynamics. IEEE Transactions on Control Systems Technology, 7(4):487-493, 1999.

[34] A. Kong, J. S. Liu, and W. H. Wong. Sequential imputations and Bayesian missing data problems. J. Amer. Stat. Assoc., 89(425):278-288, 1994.

[35] J.S. Liu. Metropolized independent sampling with comparison to rejection ampling and importance sampling. Statistics and Computing, 6:113-119, 1996.

[36] P-J. Nordlund and F. Gustafsson. Sequential monte carlo filtering techniques applied to integrated navigation systems. In Proc. of the American Control Conference, Arlington, Virginia, U.S.A, June 2001.

[37] M.K. Pitt and N. Shephard. Filtering via simulation: Auxiliary particle filters. Journal of the American Statistical Association, 94(446):590-599, June 1999.

[38] C. Rago, P.Willett, and R.Streit. A comparison of the JPDAF and PMHT tracking algorithms. In IEEE Conference on Acoustics, Speech and Signal Processing, volume 5, pages 3571-3574, 1995.

[39] B.D. Ripley. Stochastic Simulation. John Wiley, 1988.

[40] S. Rohr, R. Lind, R. Myers, W. Bauson, W. Kosiak, and H. Yen. An integrated approach to automotive safety systems. Automotive engineering international, September 2000.

[41] D.J Salmond, D. Fisher, and N.J Gordon. Tracking in the presence of intermittent spurious objects and clutter. In SPIE Conf. on Signal and Data Processing of Small Tragets, 1998.

42] Blackman S.S and Popoli R. Design and analysis of modern tracking systems. Artech House, Norwood, MA, 1999.

[43] S. Thrun, D. Fox, F. Dellaert, and W. Burgard. Particle filters for mobile robot localization. In A. Doucet, N. de Freitas, and N. Gordon, editors, Sequential Monte Carlo Methods in Practice. Springer-Verlag, 2001.

[44] O. Wijk. Triangulation Based Fusion of Sonar Data with Application in Mobile Robot Mapping and Localization. PhD thesis, Royal Institute of Technology, 2001. 